\title{
Einmaliger schweizerisch-europäischer Kongress für Innere Medizin
}

\section{Arnaud Perrier}

SGIM-Tagungspräsident und Präsident des wissenschaftlichen Komitees des ESCIM 2014
Korrespondenz:

SGIM

Solothurnerstrasse 68

CH-4002 Basel

info[at]sgim.ch
Vom 14. bis 16. Mai 2014 findet in Genf unter der Schirmherrschaft der Schweizerischen Gesellschaft für Allgemeine Innere Medizin (SGIM) und der European Federation of Internal Medicine (EFIM) der erste gemeinsame schweizerisch-europäische Kongress für Innere Medizin statt.

\section{Die Idee des Kongresses}

Die Allgemeine Innere Medizin hat in der Schweiz sowohl in der ambulanten Praxis als auch in den Spitälern einschliesslich Universitätsspitälern eine sehr grosse Tradition. Dass eine gemeinsame Basisweiterbildung für Innere Medizin am Spital sowohl für angehende Allgemeinmediziner als auch für angehende Allgemeininternisten existiert, ist praktisch einzigartig in Europa. Zur Förderung dieses Modells und um von den Erfahrungen anderer zu profitieren, pflegt die SGIM seit vielen Jahren Beziehungen zur European Federation of Internal Medicine (EFIM). Die EFIM umfasst 33 nationale Gesellschaften für Innere Medizin mit insgesamt etwa 33000 Mitgliedern. Präsident des EFIM-Vorstands war zudem Dr. Werner Bauer, der gleichfalls Präsident der SGIM war. Die Idee, die EFIM an der Jahrestagung der SGIM teilhaben zu lassen, um so unseren Publikumskreis zu erweitern und den Austausch zwischen Kollegen verschiedener europäischer Länder zu fördern, lag somit klar auf der Hand.

\section{Ersetzt der Kongress die SGIM-Jahrestagung?}

2014 wird es keine weitere SGIM-Jahresversammlung geben. Es ist unsere Jahrestagung, die aber durch die Teilnahme unserer europäischen Partner noch bereichert wird.

\section{Warum «Together»?}

Nur «miteinander» können Medizin und insbesondere die Allgemeine Innere Medizin heutzutage praktiziert werden:

- Miteinander: Allgemeininternisten und Spezialisten;

- Miteinander: Ärzte, Pflegepersonal und weitere Gesundheitsfachpersonen;

- Miteinander: medizinisch-pflegerisches Netzwerk der Klinik und öffentliche Gesundheitsversorgung;

- Miteinander: Sozialarbeiter und Verwaltungs-Verantwortliche in Quartieren und Gemeinden.

Die Liste liesse sich noch lange fortsetzen. Der Übergang von ärztlicher zu interdisziplinärer Versorgung findet gegenwärtig zögerlich statt. Wir beobachten, wie spezialisierte Versorgungsnetze und klinische Behandlungspläne entstehen, die häufig auf eine einzige Pathologie wie beispielsweise «Diabetes mellitus»

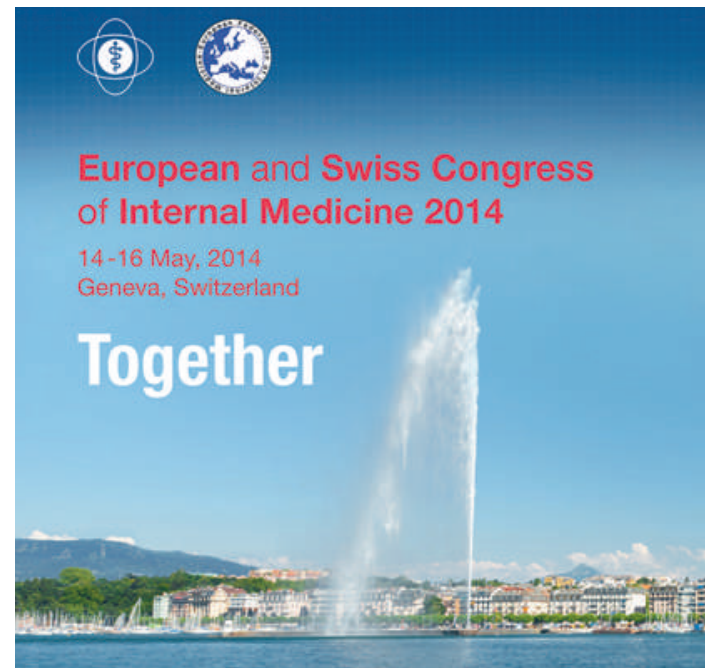

ausgerichtet sind. Die Wirklichkeit unserer Patienten wandelt sich indes schneller als unsere Modelle. Heute wird Multimorbidität zur Regel, und dies trifft auch bereits auf jüngere Patienten zu. Die Entwicklung muss also weiter fortschreiten in Richtung einer integrierten Versorgung.

Deshalb haben wir gemeinsam mit Frau Prof. Sabina de Geest, Professorin für Pflegewissenschaft, einen kompletten Halbtag zum Thema «Integrated Care» ausgearbeitet, der sich auf mehrere konkrete Beispiele aus Europa und der Schweiz stützt. Es geht nicht nur darum, Ärzte, Pflegepersonal und weitere Gesundheitsfachpersonen einzubinden, sondern auch darum, das Versorgungsnetz von der öffentlichen Gesundheitsversorgung bis zum Spital und umgekehrt zu organisieren. Eine weitere Neuerung dieses Kongresses besteht darin, dass wir Studierende der Medizin und der Pflegewissenschaft zu jenem Tag, an dem diese Veranstaltung stattfindet, kostenlos einladen. Wir hoffen, so nicht nur die Aufmerksamkeit der Studierenden auf diese neuen Modelle zu richten, sondern möchten ihnen auch ermöglichen, an weiteren Veranstaltungen teilzunehmen ... miteinander.

\section{Warum in Genf?}

Aus praktischen Gründen fand die SGIM-Jahresversammlung seit Jahren nicht mehr in Genf statt, da die Stadt sehr dezentral liegt. Demgegenüber hat das weltoffene Genf nicht nur eine internationale Ausstrahlung, sondern befindet sich auch zentral in Europa und ist gut erreichbar. Genf ist eine lebendige, charmante Stadt mit reichem historischen und architektonischen Erbe sowie einer herrlichen Landschaft. 


\section{Schweizerische Gastgesellschaften am ESCIM 2014}

- Schweizerische Akademie für Psychosomatische und Psychosoziale Medizin

- Schweizerische Fachgesellschaft für Geriatrie

- Schweizerische Gesellschaft für Allgemeinmedizin

- Schweizerische Gesellschaft für Hämatologie

- Schweizerische Gesellschaft für Klinische Pharmakologie und Toxikologie

- Schweizerische Gesellschaft für Nephrologie

- Schweizerische Gesellschaft für Infektiologie

- Schweizerische Hypertonie-Gesellschaft

- Schweizerische Gesellschaft für Physikalische Medizin und Rehabilitation

- Schweizerische Gesellschaft für Notfall- und Rettungsmedizin

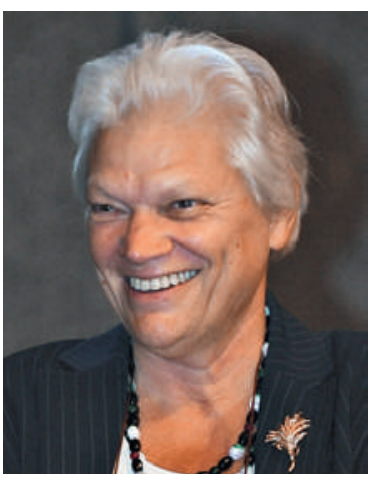

Prof. Dr. med. Maria Domenica Cappellini, Präsidentin der EFIM.

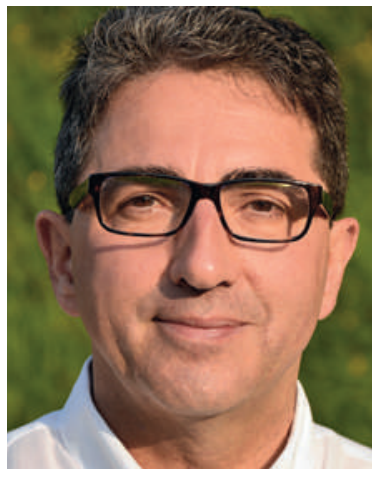

Prof. Dr. med. Arnaud Perrier, SGIM-Tagungspräsident 2014.

\section{An wen richtet sich der Kongress?}

Unser Ziel ist, dass der geplante ESCIM-Kongress ein äusserst breites Publikum anspricht. Er richtet sich somit an alle Allgemeininternisten, unabhängig davon, wo sie praktizieren (Praxis oder Spital), und unabhängig von deren Ausbildungsniveau (Facharztweiterbildung oder Fortbildung) und ihren Interessenschwerpunkten (wissenschaftliche Inhalte oder qualitativ hochwertige Fortbildung). Der Kongress richtet sich zudem an Fachärzte für Allgemeine Innere Medizin; einige der jeweiligen Fachgesellschaften sind wie üblich Gastgesellschaften am Kongress (siehe Informationskasten). Angesprochen sind darüber hinaus auch Studierende der Medizin und der Pflegewissenschaft.

\section{Das Programm}

Für 2014 haben wir die Formate gewählt, mit denen die Teilnehmer der SGIM-Jahresversammlung 2013 bereits vertraut sind. Die Plenarsitzungen bieten eine breite Themenpalette mit mehreren parallel stattfindenden Veranstaltungen. Die einzige Besonderheit des ESCIM 2014 besteht darin, dass die Plenarsitzungen auf Englisch abgehalten werden, so dass die Teilnahme für jeden möglich ist (bei den Workshops und Learning Centers dagegen ist dies nicht durchgängig der Fall). Das vollständige Programm kann auf der Internetseite des Kongresses unter www.escim2014.org abgerufen werden.

In den Trend Lectures sollen vielfältige Themenbereiche unseres Berufsfelds, die von allgemeiner Tragweite sind, zur Sprache kommen. Wir freuen uns, auf die Teilnahme hochkarätiger Redner aus Nordamerika und Europa zählen zu dürfen, wie beispielsweise Zeke Emanuel von der Universität von Pennsylvania, der sich mit uns Fragen zu ethischen Aspekten der Rationierung im Gesundheitswesen stellt; David Bates vom Brigham and Women's Hospital in Boston referiert über die Frage, ob das wachsende Qualitätsbewusstsein an der Lebenswirklichkeit unserer Patienten etwas ändert; Sanjay Saint aus Ann Arbor wird zu Bias in der klinischen Abklärung von Diagnosefehlern sprechen; sowie Jacques Cornuz von der SGIM und Erdal Alkalin von der EFIM, die uns über den aktuellen Stand der Choosing wisely-Aktivitäten in der Schweiz und in Europa berichten werden. Louis Loutan aus Genf wird über Gesundheit und Migration, ein wichtiges, aktuelles Thema, sprechen. Sophia de Rooij aus den Niederlanden wird uns einladen, mögliche gangbare Wege für den wachsenden Bedarf in der Versorgung hochbetagter Menschen zu reflektieren. Victor Montori von der Mayo Clinic wird über die wachsende Beteiligung unserer Patienten als Akteure im Gesundheitssystem sprechen. Und über seine Erfahrungen mit der Pflegestation von morgen wird uns William Ghali, ein namhafter Klinikexperte aus Calgary, berichten.

Die Updates ermöglichen eine Bestandesaufnahme über Neuerungen in allen grossen Fachbereichen der Allgemeinen Inneren Medizin, mit besonderer Berücksichtigung der Bereiche Geriatrie und Notfallmedizin. Die Symposien werden äusserst facettenreich sein und sehr viele verschiedene Themenbereiche behandeln, vom Management des kardiovaskulären Risikos über die Fortschritte und Grenzen der modernen Bildgebung bis hin zu Genetik für den Internisten, Women's health, zielgerichteten Therapien bei Krebs und Autoimmunerkrankungen sowie Palliative Care. Die How-to-Sessions liefern einen Überblick zur aktuellen, evidenzbasierten Versorgung klinischer Probleme und häufiger Erkrankungen wie beispielsweise Vorhofflimmern, Herzinsuffizienz, COPD und Diabetes, aber auch Schwindel und Schlaflosigkeit. Nicht zu vergessen einige klinische Perlen.

Die Sonderveranstaltung $\mathrm{zu}$ Integrated Care wurde bereits erwähnt. Ergänzt sei noch der Halbtag, der von den Young Internists organisiert wird, einer sehr aktiven Gruppierung innerhalb der EFIM, deren Schweizer Sektion vor einem Jahr gegründet wurde.

Das Programm ist stets äusserst ansprechend und ermöglicht Internisten in Weiterbildung aus allen Ländern, sich zu begegnen und auszutauschen.

Und schliesslich gibt es wie gewohnt die Workshops und Learning Centers. Geplant sind mehr als 90 Workshops, die auf Französisch, Deutsch oder Englisch abgehalten werden. Die Learning Centers werden ebenfalls in mehreren Sprachen angeboten und sind dieses Jahr stark an der Ultraschalldiagnostik für Internisten, an der Einschätzung des Notfalls und der Patientenschulung ausgerichtet. Dieses Jahr sind zudem die bisherigen zusätzlichen Kosten für die Workshops und die Learning Centers bereits im Kongresseintritt inbegriffen.

\section{Der wissenschaftliche Teil}

Wir erwarten eine sehr grosse Anzahl an Abstracts aus der Schweiz und ganz Europa. Es wird also qualitativ anspruchsvolle Vortragsveranstaltungen und sehr viele Poster geben. Junge Internisten mit Interesse an der Forschung werden hier Gelegenheit haben, ihre Arbeiten zu präsentieren und sie mit ihren Kollegen zu teilen. Die besten Arbeiten werden wie in jedem Jahr prämiert.

\section{Schlussbemerkung/Einladung}

ESCIM 2014 wird ein Kongress mit ausserordentlich vielen Gelegenheiten zum Lernen, Teilen und zur Begegnung sein. Mit Frau Prof. Maria Domenica Cappellini, Präsidentin der EFIM, und dem gesamten wissenschaftlichen Komitee des Kongresses laden wir Sie ganz herzlich zu dieser Zusammenkunft in Genf ein. 